\title{
ANÁLISIS DE LA POLÍTICA EXTERIOR DE XI JINPING HACIA EL SUDESTE DE ASIA: ESTRATEGIAS, INTERESES Y DIMENSIONES
}

\author{
ANALYSIS OF XI JINPING'S FOREIGN POLICY TOWARDS \\ SOUTHEAST ASIA: STRATEGIES, INTERESTS, AND \\ DIMENSIONS
}

\section{Florencia Rubiolo ${ }^{1}$ \\ ORCID: 0000-0002-5669-7332}

Virginia S. Busilli ${ }^{2}$

ORCID: 0000-0002-6305-8010

\author{
Milton E. Escobar ${ }^{3}$ \\ ORCID: 0000-0002-2473-2236
}

\section{RESUMEN}

El presente trabajo analiza la política exterior de Xi Jinping hacia el Sudeste de Asia, focalizando en las dimensiones político-estratégica y económica. Se toma como premisa, coincidiendo con una extensa línea de estudios, que sus acciones y estrategias muestran mayor visibilidad y dinamismo que las ejecutadas por sus antecesores, tanto a nivel regional como global. A partir de la revisión bibliográfica se identifica que China combina, por un lado, una política exterior asertiva en cuestiones de soberanía territorial, como la defensa de su soberanía marítima en el Mar de China Meridional y; por el otro, la integración económica y comercial basada en la interdependencia y el multilateralismo, a través del fortalecimiento de los lazos regionales en el marco de la ASEAN+3, el Área de Libre Comercio China-ASEAN y la Belt and Road Initiative. Este artículo se centra en la complementariedad de las acciones e iniciativas de la política exterior china hacia la región, a partir del desarrollo de una estrategia holística de proyección global, basada en la consolidación de un espacio regional de poder y la consecuente construcción de liderazgo.

1 Universidad Católica de Córdoba, Centro de Investigaciones y Estudios sobre Cultura y Sociedad Consejo Nacional de Investigaciones Científicas y Técnicas (CIECS-CONICET), Argentina. Doctora en Relaciones Internacionales, especialista en Estudios de Asia Oriental y directora del Doctorado en Relaciones Internacionales de la Universidad Católica de Córdoba. Correo: frubiolo@gmail.com

2 Universidad Católica de Córdoba, Centro de Investigaciones y Estudios sobre Cultura y Sociedad - Consejo Nacional de Investigaciones Científicas y Técnicas (CIECS-CONICET), Argentina. Doctoranda en Estudios Sociales de América Latina y Becaria doctoral de CONICET. Correo: virginia.busilli@gmail.com

3 Universidad Católica de Córdoba, Facultad de Ciencia Política y Relaciones Internacionales, Argentina. Investigador y profesor, Magíster en Política y Gestión Universitaria por la Universidad de Barcelona. Correo: escobarmilton@gmail.com

Florencia Rubiolo, Virginia S. Busilli y Milton E. Escobar 
Palabras clave: Política exterior china; asertividad; ASEAN; Mar de China Meridional; Nueva Ruta de la Seda.

\section{ABSTRACT}

This work analyzes Xi Jinping's foreign policy towards Southeast Asia, focusing on the political-strategic and economic dimensions. The initial assumption is that its actions and strategies show greater visibility and dynamism than those carried out by its predecessors, both regionally and globally.

From the literature review, it is identified that China combines, on the one hand, an assertive foreign policy in matters of territorial sovereignty such as the defense of its maritime sovereignty in the South China Sea and; on the other, economic and trade integration based on interdependence and multilateralism, through the strengthening of regional ties within the framework of ASEAN + 3, the China-ASEAN Free Trade Area and the Belt and Road Initiative.

This article focuses on the complementarity of the actions and initiatives of Chinese foreign policy towards the region, starting from the development of a holistic strategy of global projection, based on the consolidation of a regional space of power and the consequent construction of leadership.

Keywords: Chinese foreign policy; assertiveness; ASEAN; South China Sea; Belt and Road Initiative (BRI).

\section{Introducción}

El Sudeste de Asia (SEA) se constituye, desde tiempos antiguos, en un espacio de proyección del poder chino y, desde hace cuatro siglos, en un escenario de competencia entre potencias extra regionales. El rol que han jugado los pequeños Estados de esta región en las confrontaciones internacionales que tuvieron como escenario el Este de Asia ha sido, tradicionalmente, el de balanceadores (Arase, 2016). Tanto en la dimensión político-estratégica como en la económica, han prevalecido las disputas por espacios de poder que, aún hoy, definen la lógica de relaciones en la región.

En este contexto, la llegada de Xi Jinping al poder favoreció la implementación de una política exterior de mayor visibilidad de parte de Beijing, pero sin fracturas con respecto a la gestión anterior. Desde su asunción como presidente en 2013, la diplomacia china adquirió rasgos más activos tanto a nivel regional como global, se alejó del bajo perfil de sus predecesores a través de la implementación de una política de logros concretos con una mayor participación en asuntos globales y regionales (Nie, 2016; Yan, 2014; Zhang, 2015a). El Sudeste de Asia se convierte en una región central dentro de esta redefinida estrategia, tanto por su ubicación geográfica, como por los consolidados lazos de interdependencia económica que la vinculan.

Desde comienzos del siglo XXI, se observa una progresiva consolidación de China y su estatus de potencia global. Esto ha suscitado un debate respecto de la política más asertiva de Beijing. En la última década la diplomacia china se ha vuelto más proactiva, segura (Swaine, 2010) y asertiva (Christensen, 2012; Chang Liao, 2018; Chen, Pu y Johnston, 2014; Pu, 2017). Como sugiere Qin (2014), esta narrativa del discurso de asertividad china -o nuevo meme de 
asertividad en términos de Johnston (2013)-, se formó dentro de los círculos académicos de los Estados Unidos y se expandió a otros países originando un acalorado debate sobre las intenciones, intereses y motivaciones de Beijing. Más allá del debate en las características de la diplomacia de la RPC, el nodo central de la discusión se concentra en las intenciones detrás del ajuste de las estrategias de la política exterior, y cómo ello se vincula a la percepción de la elite del rol del China como potencia global.

Desde una visión de realismo moral, Yan (2014) discute la idea de asertividad solo como resultado de una mayor agresividad. En su lugar postula que el ajuste de política exterior hacia una mayor proactividad y visibilidad tiene una relación directa con la necesidad del país de construir credibilidad estratégica. Esto supone un acercamiento a terceros Estados, en búsqueda de apoyos -y eventualmente alianzas- fundamentales para la construcción de liderazgo global. En otras palabras, Yan (2014) comparte con la visión realista que una competencia entre potencias será inevitable, si China busca consolidarse como gran potencia global (Mearsheimer, 2014), y que ello traerá consigo la conformación de nuevas reglas que la beneficien, nuevas estructuras de gobernanza regional y global (Friedberg, 2015) y, en definitiva, un nuevo orden mundial (Yan, 2014).

Dentro de esta búsqueda de credibilidad estratégica, la región cercana es una prioridad en la política exterior de Beijing. De hecho, la llegada de Xi Jinping imprimió un nuevo dinamismo a la política exterior del país, al recuperar la periferia china como zona prioritaria. En 2013, en una visita de Estado a Indonesia, Xi mencionó la centralidad de construir una "comunidad de destino" compartida en China y ASEAN (ASEAN-China Center, 2013). Este concepto ha generado creciente atención en cuanto el primer mandatario lo convirtió en un emblema de su discurso internacional y, particularmente, regional. Ahora, como sostiene Zhang (2018), el discurso y las iniciativas económicas y políticas (particularmente la Iniciativa de la Franja y la Ruta) que buscan un acercamiento pacífico al Sudeste de Asia contrastan con las estrategias más asertivas, la preferencia por el bilateralismo y las políticas de hechos consumados que el país implementa en la dimensión político-estratégica en la región.

A partir de estas consideraciones, en el presente artículo se analizan las principales estrategias de política exterior de la República Popular China (RPC) hacia la región desde 2013 hasta la actualidad. Aunque las dimensiones que abarcan las políticas chinas hacia el Sudeste de Asia son múltiples, el trabajo se concentrará en dos: la político-estratégica y la económica. Esta elección obedece al interés por resaltar el contraste de la estrategia de política exterior china en citadas dimensiones. Por un lado, la creciente asertividad china en cuestiones de soberanía territorial y, por otro, el privilegio dado a la región del Sudeste de 
Asia en integración económica y comercial, que procura un acercamiento pacífico basado en la interdependencia y en el multilateralismo.

A modo de contextualización, se introducirán los fundamentos de la política exterior de la RPC hacia la región, identificando sus principales características y objetivos. Luego, el trabajo hará foco en el estudio de las relaciones ChinaASEAN en las dos dimensiones mencionadas: la político-estratégica, donde se destacan los conflictos en el Mar de China Meridional y el proceso de institucionalización del ASEAN Regional Forum (ARF); y la económica, donde se analizarán los casos de ASEAN+3 (APT), el ACFTA (China ASEAN Free Trade Area) y BRI (Belt and Road Initiative).

\section{Construcción del espacio de poder regional chino}

El "ascenso chino" y "la amenaza china" constituyen dos de los grandes temas de estudio sobre la política exterior de este país hoy (Kissinger, 2012; Shih y Yin, 2013). Autores como Norris (2010) y Norton (2015) sostienen que China ha desarrollado su "grand strategy" con el objetivo de lograr su estatus de potencia global. Otros autores, como Reilly (2013) y Zhang (2012) argumentan que no es posible hablar de una estrategia coherente, sino más bien de un "aprender sobre la marcha". Lo cierto es que el ascenso de China no ha resultado indiferente.

La proyección internacional de la RPC se caracterizó, desde finales de los setenta, por el mantenimiento de un bajo perfil y una activa participación, pero no confrontativa, en diversas regiones del globo y en el plano multilateral. Ahora bien, desde la crisis financiera global de 2008, la diplomacia china ha tomado una dirección más asertiva y confiada en la arena internacional, lo cual ha intensificado las preocupaciones de los países vecinos respecto al ascenso de China (Greitens, 2013).

En línea con lo anterior y desde su arribo a la presidencia, Xi Jinping expuso su visión de lo que sería el núcleo de la ideología guía de su mandato (Vélez Serrano, 2018). Con el eslogan del "sueño chino", Xi hizo referencia, en su discurso de inauguración del nuevo liderazgo chino en 2012, a la necesidad de lograr el rejuvenecimiento de la nación, con el objeto de que esta "se pare con mayor firmeza y poderosamente entre todas las naciones del mundo y hacer una mayor contribución a la humanidad" (Vélez Serrano, 2018, p. 16). Esta constituye una de las múltiples referencias a la necesidad de que el país asuma un rol más activo en el sistema internacional (Zhang, 2015). En este sentido, en el marco de la 70a sesión de la Asamblea General de Naciones Unidas en 2015, Xi destacó la 
importancia de un "entorno internacional pacífico, de un orden mundial estable y de la comprensión, apoyo y ayuda entre todos los pueblos" para alcanzar el sueño del pueblo chino. Resaltó también el rol de China en este proceso, como impulsor de la paz y el desarrollo mundial (Xi, 2015). Se presentó así, ante los representantes de los países del mundo, como líder de una potencia responsable, inclusiva, pacífica y no hegemónica.

Asimismo, en otro discurso citado por Vélez Serrano (2018, p. 17), Xi también resalta la promesa de "nunca buscar la hegemonía o cometer cualquier acto de expansión". Sin embargo, cabe destacar que la salvaguarda de los intereses nacionales de la RPC ha sido un tema recurrente en los diversos discursos del mandatario. Xi ha dejado en claro que dicho "desarrollo pacífico" no implicará "el sacrificio de sus intereses fundamentales con el fin único de mantener la paz" (Vélez Serrano, 2018, p. 18). En tal sentido, en 2017, y en el marco del XIX Congreso Nacional del Partido Comunista Chino, el primer mandatario manifestó la adopción de una política exterior más desafiante, subrayando la importancia de que el país mantenga una política exterior de defensa de sus intereses legítimos (Xi, 2017).

Aunque el énfasis se mantiene en la naturaleza pacífica de la política externa del país, se puede advertir un cambio de estilo en la retórica y en la autopercepción de poder detrás de las reivindicaciones nacionales. En tal sentido, Zhang (2015) afirma que China asiste a una nueva fase de la política exterior - el "ascenso pacífico 2.0"-, en la cual, mientras Beijing todavía adhiere a su política de desarrollo pacífico para mantener un ambiente externo estable, la nueva dirección se está alejando decididamente del enfoque político de larga data de la RPC, de "esconder las propias capacidades y esperar el propio tiempo" (Zhang, 2015, p. 7-8).

La región del Sudeste de Asia tiene un rol central en este diseño de política exterior y proyección global del país, por dos razones principales: en primer lugar, el apoyo de los diez Estados que forman parte de la ASEAN resulta necesario para cualquier potencia con aspiraciones de liderazgo regional y aporta una plataforma clave para cualquier iniciativa regional. En segundo lugar, porque pone a prueba la capacidad de respuesta de China frente las necesidades de los países que la apoyan (Ba, 2014). En este sentido, Chi Zhang (2013, p. 6-7) identifica tres períodos de acercamiento desde la fundación de la República Popular. A través de ellos puede observarse un creciente compromiso e interés de Beijing con su periferia:

- el primer período, entre 1949 y 1978, se caracterizó por respuestas pasivas de China frente a las situaciones internacionales, 
- el siguiente período, comprendido entre 1978-1989, estuvo marcado por un ajuste y moderación, guiados por el foco de Beijing en su proceso de reformas económicas. Durante estos años primó un acercamiento cooperativo, pacífico y estable, con el objetivo de fortalecer los vínculos regionales, finalmente

- $\quad$ el tercer y último período, desde 1990 en adelante, se identifica un manejo activo, donde la República Popular propone activamente asociaciones con los países vecinos, fomentando vínculos pacíficos de largo plazo.

Autores como Zhang (2012) destacan que este proceso ha sido de naturaleza exploratoria, evolutiva y adaptativa. De este modo, se observa la continuidad de la política exterior de la gestión de Hu Jintao en el mandato de Xi Jinping, sino la evolución hacia un carácter prioritario en la agenda china.

A pesar de la búsqueda activa por establecer relaciones estables y a largo plazo con los países vecinos, el Este de Asia representa, para Beijing, importantes desafíos. Uno de los principales es el recelo que genera su crecimiento económico entre sus vecinos (Zhang, 2013). Sobre el trasfondo de esta percepción de amenaza y la emergente asertividad territorial -en particular en el Mar de China Meridional (MCM), la élite dirigente china procuró, a través de diferentes plataformas discursivas, dejar en claro las intenciones pacíficas del país en la región. El interés chino en asegurar la paz y la estabilidad en su entorno es una condición necesaria para su desarrollo económico y este, a su vez, para el mantenimiento en el poder del Partido Comunista (Delage, 2015). El funcionamiento de la economía ha sido sin duda un factor de legitimidad del régimen desde el inicio del proceso de modernización del país (Rocha Pino, 2020).

Como sostienen Delage (2015) y Callahan (2016), la consolidación de la influencia china en Asia es esencial para su ascenso en el sistema internacional. El afianzamiento de dicha influencia conlleva el esfuerzo chino por reducir el diferencial de poder con los Estados Unidos, uno de los principales desafíos para Beijing (Zhang, 2013). Desde la perspectiva china, Estados Unidos es la clave del enfrentamiento con sus vecinos del Sudeste de Asia y el principal factor que complica su estrategia hacia Japón, además del tradicional obstáculo que representa para la unificación de Taiwán (Delage, 2015).

En este sentido, la creciente asimetría en términos de poder económico, diplomático y estratégico otorga a la RPC una importante capacidad de gobernanza a nivel regional y subregional; ello ha generado en los países vecinos desconfianza y temor respecto de las consecuencias de su ascenso como gran potencia. Más aún cuando la salvaguarda de los denominados "intereses centrales" de la 
RPC pasa a ser una temática central en numerosos discursos de Xi Jinping. El concepto tiene una definición específica dentro de la tradición política china, de acuerdo con Nie (2016, p. 428):

Los intereses centrales constituyen una parte, la más relevante, de los intereses nacionales (seguidos por los vitales y los principales). Los intereses centrales son esencialmente no negociables por naturaleza, y para China estos incluyen temas como la soberanía de Taiwán, Tibet y Xinjiang.

En esta línea, el interés nacional puede definirse como el conjunto de objetivos diseñados y buscados por los responsables políticos centrales para mejorar la utilidad material y la ideación de valores del país en su conjunto (Zhang, 2012, p. 3). Ahora bien, aunque el concepto responde a intereses estables de la RPC, se observa que su contenido y jerarquía han sufrido vaivenes que responden a las orientaciones de sus líderes, el contexto nacional y externo, y las coyunturas políticas y económicas.

A partir de 2003, se identifica una mayor referencia al término dentro del discurso político de los funcionarios chinos. En 2010, Dai Bingguo, consejero de Estado a cargo de los asuntos externos de la RPC, definió los intereses centrales del país de la siguiente manera y en este orden: "mantener el sistema político y la seguridad estatal; luego la soberanía estatal y la integridad territorial; y tercero el desarrollo estable de la economía y la sociedad" (Dai Bingguo, 2010). Aunque no se hace una referencia explícita a territorios fuera de los mencionados previamente, los países del Sudeste de Asia que comparten reclamos territoriales con China en el Mar de China Meridional (Brunei, Filipinas, Malasia y Vietnam) han percibido esta ampliación de los intereses centrales como una advertencia respecto de las intenciones de Beijing en las disputadas aguas.

En suma, la redefinición de la política exterior china durante el gobierno de $\mathrm{Xi}$ muestra una faceta más enérgica y reivindicativa, aunque sin quebrar con los principios generales de acercamiento a la región de su antecesor: desde la premisa de "paz y desarrollo" profundizar la cooperación ganar-ganar y lograr que el crecimiento chino se traduzca en mayores beneficios para sus vecinos (Zhang, 2013). El creciente poder relativo ha dado a los dirigentes una autopercepción de superioridad que, de alguna manera, faculta al país a redefinir sus espacios de intervención a nivel internacional, así como la extensión de sus reclamos territoriales, con el objetivo de atender sus intereses nacionales de crecimiento y seguridad en un ambiente regional estable y controlado (Li, 2009; Yahuda, 2013). Este escenario, lejos de calmar la ansiedad de los países vecinos, enciende las alarmas respecto de las intenciones de Beijing y de la forma que tomaría un liderazgo chino en la región. Ello se puede ver reflejado en una serie 
de movimientos contrarios a los intereses de la RPC a largo plazo, como los cambios en la política de seguridad japonesa, la asociación estratégica de Japón con India y con Australia (y la de estos dos últimos países entre sí), la creciente unidad de la ASEAN con respecto a la RPC, la proliferación de acuerdos bilaterales de seguridad entre potencias intermedias o el papel de Estado Unidos, cuya protección es deseada por numerosos Estados de la región (Delage, 2013).

En contraste, el liderazgo chino iniciado por Xi puso también el énfasis en la expansión y proliferación de herramientas de poder blando y en la consolidación de la interdependencia económica en el Este de Asia, con especial foco en el Sudeste. Conceptos como la "diplomacia de la periferia" y la creación de una "comunidad de destino" están orientados a reforzar la idea del compromiso chino con las aspiraciones de desarrollo económico y seguridad de la región y disminuir la percepción de amenaza, a través de la articulación multilateral y la cooperación económica intraasiática.

\section{La dimensión político-estratégica en la relación con el SEA: Intereses y lógicas de enfrentamiento}

En 2014 Xi afirmó que la seguridad en Asia es un asunto que concierne solo a los Estados asiáticos, y que las alianzas militares representan la "mentalidad de Guerra Fría" y de juegos de "suma cero", refiriéndose, en particular, a las alianzas defensivas de los Estados Unidos y sus aliados en la región (Arase, 2016). Enfatizó, asimismo, que la paz debe basarse en el desarrollo económico cooperativo y mutuamente beneficioso.

El Foro Regional de ASEAN y el conflicto sobre las islas del Mar de China Meridional serán los dos ejes sobre los que se centrará el presente análisis, como las dos principales arenas de cooperación estratégica de estos actores, que han involucrado paulatinamente a China en negociaciones multilaterales con el Sudeste de Asia. A su vez, en esta problemática regional se pone de manifiesto más claramente hasta qué punto la RPC está dispuesta a someter el tratamiento y la resolución de conflictos regionales a ámbitos multilaterales. 


\subsection{Implicancias de la asimetría en el conflicto en el Mar de China Meridional}

El Mar de China Meridional -de acuerdo con la denominación más extendida- se encuentra emplazado en la región del sudeste de Asia y bordea el sur de China. Sus aguas albergan más de 400 islas, arrecifes y bancos de arena, y numerosos archipiélagos, entre los que se destacan las islas Spratly y las Paracel. El conflicto por el territorio marítimo y las islas comprende, actualmente, a seis partes -China, Filipinas, Vietnam, Malasia, Brunei y Taiwán- que reclaman, de manera total o parcial, el territorio (Rubiolo, 2020).

Los intereses que subyacen en las reivindicaciones se basan, en parte, en las reservas de hidrocarburos que tiene el territorio, así como de otros recursos naturales, principalmente ictícolas. Sin embargo, lo que distingue al área es su ubicación estratégica como ruta de transporte marítimo comercial. Entre los puntos de acceso que conectan esta zona, el de mayor tránsito de bienes y recursos es el estrecho de Malacca, situado entre Malasia, Indonesia y Singapur. En la actualidad, se constituye en la segunda vía de comercio marítimo más transitada del mundo, después del estrecho de Dover.

China es el actor que reivindica la mayor parte: reclama el $80 \%$ del territorio, incluyendo archipiélagos y aguas circundantes. En 2009, la RPC presentó, ante la Comisión de Límites de la Plataforma Continental de las Naciones Unidas, un mapa donde, para sorpresa de los demás demandantes, especifica que la porción de territorio que reclama se incluye dentro de lo que se denomina la "línea de nueve puntos". El territorio allí definido se superpone con los reclamos de los demás Estados costeros y plantea que las aguas y recursos en ellas contenidos también corresponden a China por derecho histórico. La reacción de los Estados ribereños fue, de inmediato, el repudio. En una reacción más contundente, Filipinas, en el mandato de Benigno Aquino, presentó en 2013 una denuncia ante el Tribunal Internacional del Derecho del Mar (ITLOS) contra la "línea de nueve puntos" y las acciones chinas dentro de la zona económica exclusiva de Filipinas (Baviera, 2016). Aunque el fallo internacional fue favorable a Filipinas, Beijing se negó a reconocer la legalidad de este y la ASEAN mantuvo una postura neutral, para evitar la confrontación con su gigante vecino.

Las acciones de la RPC han adquirido un tenor más contundente en términos militares y políticos desde 2009 en adelante. Algunas acciones, en este sentido, fueron el incidente con un buque de investigación de energía de Filipinas cerca de Reed Bank en 2011; los cortes de cables de exploración sísmica del buque vietnamita Binh Minh 2 en 2012 o el bloqueo por parte de China a un buque de la Armada de Filipinas en Scarborough Shoal en 2010 (Miks, 2012). 
Finalmente, el caso más reciente fue el despliegue chino, en mayo de 2014, de su plataforma petrolífera de aguas profundas, Haiyang Shiyou 981, en las aguas de las islas Paracel, también reclamada por Vietnam (Lye y Ha, 2018). Esta política de hechos consumados contrasta notoriamente con el discurso político de desarrollo pacífico y la política de poder blando que Beijing, en paralelo, está desplegando en el Sudeste de Asia.

Frente a este creciente asertividad, los países del área también comenzaron a procurar alternativas para garantizar su seguridad, entre los que se destaca la política defensiva de Vietnam de establecimiento de diálogos estratégicos y de defensa con poderes regionales y extrarregionales (con Japón en 2010, con Corea del Sur y el Reino Unido en 2018); la firma del "Memorando de entendimiento en defensa y la cooperación financiera para fortalecimiento de las capacidades militares" con Estados Unidos (Hiep, 2015).

En este contexto, el rol de Estados Unidos cobra mayor relevancia a partir de 2010, luego del discurso de Hillary Clinton en el marco de ARF, que subrayó la centralidad de la libertad de navegación, refiriéndose específicamente al Mar de China Meridional ${ }^{4}$. A partir de 2011, con el lanzamiento de la estrategia Rebalance to Asia, Estados Unidos decidió duplicar sus esfuerzos diplomáticos, económicos y estratégicos para fortalecer su presencia en el Asia Pacífico, en parte como respuesta a las demandas de ASEAN, pero también como reafirmación de su poderío frente a la expansión de China en diversas dimensiones de poder regional (Saunders, 2013).

El renovado compromiso norteamericano como garante de la seguridad de los países de la región y, particularmente, de los que comparten la disputa marítima con China, es contemplado desde la cúpula de Beijing como una estrategia de contener su expansión. A su vez, el argumento de libertad de navegación se interpreta como un subterfugio para justificar una ampliación de las actividades militares en el área, inscripto en una lógica de competencia por espacios de poder regional (Yang y Li, 2016).

Las instancias multilaterales de negociación, sobre las que se han asentado los principales esfuerzos de ASEAN para disminuir la incertidumbre en el marco del conflicto, pusieron en evidencia la escasa predisposición de los líderes de la RPC para comprometerse en procesos de esta naturaleza (Hoang, 2019; Severino, 2010). La primera de ellas fue la negociación por la "Declaración sobre

4 "The United States, like every nation, has a national interest in freedom of navigation, open access to Asia's maritime commons, and respect for international law in the South China Sea. We share these interests not only with ASEAN members or ASEAN Regional Forum participants, but with other maritime nations and the broader international community" (Clinton, 2010). 
la conducta de las partes en el Mar de China Meridional (DOC)", firmada en Camboya en 2002. Luego de tres años de negociaciones, el resultado, tras las numerosas objeciones del gobierno de la RPC, fue una declaración sin carácter vinculante para las partes y con falencias para la definición concreta de las actividades restringidas en el territorio en disputa. En este sentido, uno de los principales obstáculos a la implementación fue la ambigüedad en la delimitación de los reclamos territoriales de cada país, particularmente de los territorios incluidos en la línea de nueve puntos que China reclama. En consecuencia, la indefinición de las áreas disputadas llevó a la imposibilidad de definir en qué zonas se aplicaría el autocontrol en las actividades de los países que se plantea en el párrafo 5 de la $\mathrm{DOC}^{5}$ (ASEAN, 2012).

En 2011, ASEAN y China llegaron a un nuevo acuerdo sobre los lineamientos para implementar la DOC de 2002. Sin embargo, como postula Fravel (2011), este nuevo acuerdo tuvo limitaciones, debido a que la DOC no era un documento vinculante en sí mismo, sino un primer paso hacia la elaboración de un código de conducta; además la DOC no aborda los conflictos de soberanía en sí mismos, sino que busca disminuir las tensiones; y los lineamientos se limitaban a espacios de intercambio de información y coordinación de temas amplios (medioambiente, seguridad marítima, operaciones de rescate y crimen transnacional) no relacionados con las tensiones derivadas de la superposición de los reclamos territoriales y las acciones de ocupación de facto.

De manera más reciente, ASEAN promovió la negociación de un Código de Conducta de las Partes (COC), como mecanismo para promover mayor previsibilidad en el comportamiento de los Estados, con especial foco en China. El primer borrador fue presentado por los diez Estados de ASEAN y China en agosto de 2018 y se encuentra en negociación. A pesar de las expectativas iniciales, el documento difícilmente pueda concluir en un éxito diplomático. La ASEAN tiene un escaso margen de negociación (Roberts, 2018), mientras los líderes chinos han mostrado inicial reticencia, y luego una tibia participación en las conversaciones (Storey, 2018). No obstante, la participación china refleja el interés del gobierno de mostrar un cierto grado de compromiso con el multilateralismo regional, como herramienta de poder blando para disminuir la percepción de amenaza que su poderío alienta en sus vecinos, resguardando al mismo tiempo sus intereses nacionales ( $\mathrm{Li}, 2009)$.

5 Traducción propia del original en inglés: "Las Partes se comprometen a ejercer autocontrol en la realización de actividades que complicarían o intensificarían las disputas y afectarían la paz y la estabilidad, incluidas, entre otras, abstenerse de la acción de habitar en las islas, arrecifes, cardúmenes, cayos y otras características actualmente deshabitadas, y a manejar sus diferencias de manera constructiva" (ASEAN, 2012). 
Algunos autores plantean que uno de los principales desafíos para Bejing en este conflicto es mantener el balance entre la estabilidad regional y garantizar sus intereses en la disputa (Yang y Li, 2016). China ha mostrado un mayor compromiso en el diálogo y la cooperación con los países de ASEAN, pero su principal estrategia hoy se asienta sobre una política de hechos consumados: la expansión de medidas de patrullaje y control de los territorios ocupados, el mantenimiento del status quo y la negativa a avanzar en procesos de resolución multilateral del tema de la soberanía, apostando a una estrategia de retraso que le permita ganar tiempo para fortalecer sus capacidades frente a los demás países costeros (Fravel, 2011).

\subsection{El Foro Regional de ASEAN y el desafío del multilateralismo para Beijing}

El ARF es una estructura de diálogo estratégico multilateral a la cual se dio inicio en la reunión anual de los ministros de relaciones exteriores de los países de ASEAN en Singapur, en julio de 1993 (Leifer, 1995). El Foro incluye, en la actualidad, a los diez países de ASEAN y a los diecisiete socios: Australia, Bangladesh, Canadá, China, Estados Unidos, India, Japón, Mongolia, Nueva Zelanda, Pakistán, Papua Nueva Guinea, la República Democrática de Corea, la República de Corea, Rusia, Sri Lanka, Timor Oriental y la Unión Europea. El Foro Regional se convirtió en una pieza central en la arquitectura de seguridad regional de ASEAN, a partir de la creación de un espacio de discusiones sobre arreglos regionales de seguridad, el desarrollo de instituciones "blandas" relacionadas principalmente con estructuras normativas informales, y con un creciente énfasis en preocupaciones de seguridad no tradicionales (Kraft, 2017). Además, el Foro puso de manifiesto la intención de los países de ASEAN de ampliar los horizontes estratégicos, abordando el problema de la seguridad regional de forma común e incluyendo a potencias extrarregionales en el diálogo multilateral (Leifer, 1994).

La conformación de ARF como organismo multilateral para la seguridad regional fue tomada con cautela por la dirigencia china. Como sostiene Kuik (2005), algunos temores radicaban en que Estados Unidos -miembro del organismo- lo utilizara como mecanismo para ampliar su influencia en la región; en que los países de ASEAN utilizaran la plataforma para internacionalizar el conflicto por las islas del MCM, y finalmente el temor a que se incluyera en la agenda de ARF el tema de Taiwán. Pero para ASEAN, la participación de Beijing en el organismo era una condición de primer orden y "un requisito fundamental para su relevancia” (Kraft, 2017, p. 603). 
La doctrina del Ascenso Pacífico, que tenía como prioridad garantizar a sus vecinos que el crecimiento de China no sería una amenaza para el bienestar económico ni para la seguridad territorial y estratégica, terminó favoreciendo una mayor participación en los procesos multilaterales para reducir la retórica de amenaza en su vecindario al crear una imagen más benigna ( $\mathrm{Li}, 2009$; Hoang, 2019).

Los gobernantes chinos evaluaron que la tendencia hacia el multilateralismo en la seguridad regional era un proceso que no podía detenerse, y del cual China no podía quedar al margen. No participar en ARF implicaría aislarse de la región y podría despertar sospechas de los países de ASEAN en un momento en que el país intentaba acercarse a sus vecinos. Por lo tanto, China optó por participar del organismo para evitar el aislamiento y para poder dar forma, desde adentro, al incipiente proceso de cooperación, de manera que no amenacen sus intereses ni su seguridad nacional (Kuik, 2005).

La postura china sobre los procesos multilaterales en la región, incluidos los vinculados con temas de seguridad, evolucionó de la cautela hacia un rol activo y de coordinación (See Seng, 2016). Esto puede apreciarse en los incentivos iniciales que llevaron a Beijing a formar parte del Foro y, luego, a participar más activamente, en particular en los procesos que involucran exclusivamente a países del Este de Asia (Kraft, 2017; Yang y Li, 2016). No obstante, su acercamiento a las instancias multilaterales, la preferencia por estrategias bilaterales y de corte realista continúa prevaleciendo (Hoang, 2019; Southgate y Khoo, 2016). Esto puede verse principalmente en áreas que se definen como más vulnerables desde la perspectiva de Beijing, como la negativa a hacer referencia a la soberanía sobre las islas del MCM en el marco de diálogos multilaterales de ARF, a discutir sobre el desarrollo en las partes del archipiélago sobre los que ejerce posesión y a darle un tratamiento formal de resolución de controversia. Su intervención en el proceso de elaboración del COC sobre el Mar de China Meridional, descrito anteriormente, refuerza esta afirmación.

\section{El espacio económico-comercial: ASEAN+3, ACFTA y BRI}

Las iniciativas que abrieron las puertas al acercamiento económico multilateral fueron principalmente dos: ASEAN +3 (APT, por sus siglas en inglés) y el ACFTA (ASEAN China Free Trade Area). Ambas pueden considerarse un antecedente multilateral de la reciente Nueva Ruta de Seda, impulsada por China y a la que se conoce como BRI (Belt and Road Initiative), que tiene 
al Sudeste de Asia como uno de sus escenarios prioritarios en el marco de una estrategia geoeconómica global.

Es necesario destacar que, si bien todas estas iniciativas apelan al multilateralismo, difieren en sus concepciones y naturaleza. Tanto el ASEAN +3 como el ACFTA tienen como protagonistas a las naciones del Sudeste de Asia exclusivamente. Asimismo, muestran una impronta recíproca, donde el fuerte dinamismo ha dependido de la voluntad política de ambas partes. La iniciativa BRI, sin embargo, es una estrategia de naturaleza estatal unilateral diseñada para responder a los objetivos de Beijing. Si bien se proyecta a múltiples actores en todos los continentes, su implementación parte de acuerdos bilaterales que se articulan en una estrategia mayor y complementan los acuerdos multilaterales en los que participa la RPC.

\subsection{ASEAN+3 y la necesidad de la cooperación económica regional}

La crisis asiática de 1997 contribuyó a un cambio en las dinámicas regionales, modificando los vínculos y las percepciones mutuas. La decisión china de no depreciar su moneda durante 1997-1998 no significó un intento de liderazgo regional, sino más bien una gestión de crisis con implicancias regionales (Yang, 2010), al evitar una cadena de devaluaciones monetarias competitivas en el Este de Asia. Por otra parte, la ayuda de China a Tailandia a Indonesia contrastó con la respuesta tardía de Estados Unidos. En este sentido, las acciones de China contribuyeron a un cambio en la percepción del tipo de rol que podría tener el Sudeste Asiático, pues hasta ese momento, los países de ASEAN habían visto a China como un país receptor de asistencia, más que un proveedor de ella ( $\mathrm{Ba}$, 2014).

En un intento de generar soluciones regionales, los países de ASEAN convocaron, en 1997, por primera vez, una reunión informal con los países del Noreste de Asia -China, Japón y Corea del Sur-. Esta reunión se constituyó en el puntapié inicial del proceso de cooperación económica regional conocido como ASEAN + 3. En sus inicios, APT tuvo dos objetivos principales: 1) limitar los efectos que la crisis financiera había generado en las economías del Este Asiático y poder prevenir próximas, por medio del diálogo y la cooperación regional y 2) evitar la marginalización económica de la región del Sudeste de Asia a través de la integración con el noreste (Wong, 2007).

La cooperación financiera se fortaleció a través de la creación del Banco Asiático de Desarrollo, en el año 2000, con el objetivo de asistir a los países de la 
región (principalmente de ASEAN) frente a dificultades relacionadas con la balanza de pagos (Haacke, 2002). En 2001, China firmó Acuerdos Swaps bilaterales con Tailandia y Malasia y, a partir de entonces, el gobierno chino ha organizado varios foros técnicos para representantes de los bancos centrales del grupo 10+3 en Beijing y Shanghai (Hernández Pedraza, 2005).

Si bien el foco de la cooperación se situó en el área financiera, a comienzos del siglo XXI la cooperación amplió su alcance y se desarrolló en múltiples niveles, como política y seguridad, crimen trasnacional, medio ambiente, turismo, seguridad alimentaria, seguridad energética, entre otros (ASEAN Secretariat Information Paper, 2017). El rol de China como impulsor de ASEAN + 3 y su creciente interés de darle un mayor alcance temático a la integración en el Este de Asia tuvieron una fuerte influencia en los avances del proceso.

Desde la cúpula de Beijing se percibía a APT como una plataforma a partir de la cual promover la cooperación en Asia Oriental, con probabilidades de expandir su influencia política y moldear el escenario estratégico regional a largo plazo (Kuik, 2005). En noviembre de 2002, China y ASEAN firmaron el Acuerdo Marco de Cooperación Económica, que entró en vigor en julio de 2003 y estableció las bases legales de cooperación y la agenda a desarrollar.

Inicialmente, China introdujo aranceles preferenciales especiales para cuatro países de ASEAN: Camboya, Laos, Myanmar y Vietnam. Por otra parte, Beijing anunció el Plan de Reducción de Deuda para Asia, en el que propuso reducir o cancelar las deudas con seis países de la región. China redujo la deuda de Camboya por un valor de 200 millones de dólares y eliminó aranceles a gran parte de sus productos. Acciones similares llevó a cabo con Laos y Myanmar, en línea con el esfuerzo de ASEAN de ayudar a sus países miembro más pobres (Hernández Pedraza, 2005). En la Cuarta Cumbre de APT, el primer ministro de China Zhu Rongji sugirió que APT debía concentrarse en algunas áreas específicas de cooperación: el desarrollo del transporte y las comunicaciones en la cuenca del río Mekong, tecnología, desarrollo de recursos humanos, agricultura y turismo (Rubiolo, 2010).

Más recientemente, y en línea con lo anterior, entre los días 12 y 14 de noviembre de 2017 se desarrolló, en Filipinas, la XXXI Cumbre de la ASEAN y sus reuniones paralelas, ASEAN +1, la XX Cumbre de ASEAN+3 y la XII Cumbre de Asia Oriental. En la Cumbre ASEAN+3 se destacó la propuesta china de avanzar hacia la construcción de una comunidad económica de Asia Oriental, a fin de potenciar la integración regional y el desarrollo común. Dado el importante dinamismo económico del Este de Asia durante las últimas décadas, este 
se vuelve un objetivo estratégico de la cooperación $10+3$, que responde a los intereses fundamentales y de largo plazo de la región (González Saez, 2017).

Durante el pasado mes de abril de 2020, y en el marco de la pandemia global, se llevó a cabo la Cumbre Especial de ASEAN+3 sobre el Covid-19. En ella se comprometieron a mantener la apertura de mercado, a firmar la Asociación Económica Integral Regional (RCEP), garantizar la circulación de bienes y servicios, especialmente materias primas y producto sanitarios, cooperar en la investigación sobre la vacuna SARS-CoV-2, así como de fármacos antivirales. También destacaron la necesidad de generar una respuesta regional a la crisis sanitaria y elaborar estrategias de recuperación económica conjuntas ( $\mathrm{La} \mathrm{Voz}$ de Vietnam, 2020). Este evento revela el avance de la integración de China con su región y profundiza no solo los lazos económicos, sino las respuestas políticas coordinadas frente a diferentes temas que afectan a los países del Este de Asia

A partir de su experiencia en APT, el multilateralismo en el área económica emerge y, en la actualidad, se constituye en un pilar dentro de la de política exterior regional de China. El establecimiento del área de libre comercio con ASEAN, al que la RPC otorgó máxima prioridad en su agenda externa a principios del siglo XXI, se inscribe en esta misma lógica.

\subsection{EI Área de Libre Comercio China-ASEAN y la consolidación de la cooperación económica regional}

La propuesta del Área de Libre Comercio fue presentada por China en 1999, durante la tercera Cumbre de APT en Manila. En medio de la recuperación económica, la activa participación de Beijing mejoró su imagen como posible líder responsable y lo convirtió en un modelo de estabilidad económica (Zhang, 2006). Como se mencionó anteriormente, fue una excelente oportunidad para demostrar a la ASEAN cuán diferente fue la actitud china frente a la crisis financiera de 1997, a diferencia del pasivo accionar de sus otros dos socios principales: Japón y Estados Unidos (Khan y Yu, 2013). El 1 de enero de 2010 ASEAN inició el acuerdo con China, transformándose en el área de libre comercio más grande del mundo, con una población de aproximadamente 1900 millones de personas (Cai, 2003).

Los motivos detrás de la conformación del ACFTA son múltiples. En términos generales, esta iniciativa es una respuesta de ambas partes a la intensificación del regionalismo económico a nivel mundial y al impacto de la crisis financiera asiática: 
- Desde el punto de vista de Beijing, el ACFTA permitía disipar los temores de ASEAN respecto de su crecimiento. Y no solo eso: el fortalecimiento de la integración económica con la ASEAN a través del ACFTA ayudaría a China a garantizar a la comunidad internacional que su ascenso pacífico no debía ser concebido como una amenaza (Khan y Yu, 2013).

- Desde el punto de vista de ASEAN, el acuerdo se convertía en una forma de asegurarse que la gigante economía china no se desarrollaría a expensas del bienestar económico de la región.

Dado que sus mayores lazos comerciales son con las economías del noreste de Asia, ASEAN buscó acercarse a esta región como medio para mejorar su posicionamiento internacional. Al mismo tiempo, procuró prevenir futuras crisis a través del fortalecimiento de la cooperación. En este contexto, China fue capaz de responder a las preocupaciones comerciales y de inversión de los países de ASEAN, incrementadas tras su ingreso a la OMC en 2001. Como se observó anteriormente, Beijing mostró su buena fe, al otorgar un trato especial y diferenciado a los países de la ASEAN, que resultó fundamental para lograr el Acuerdo Marco de 2002.

Lo cierto es que luego de más de dos décadas de integración, las relaciones bilaterales se han consolidado. A pesar de los recelos que aún despierta su ascenso, China muestra un mayor compromiso con su región. En este sentido, la interdependencia económica es clara: entre 2009 y 2019 China fue el principal socio comercial de ASEAN, mostrando índices crecientes de comercio. Mientras en 2009, las exportaciones de ASEAN con destino China ascendían a 81511 millones de dólares, en 2019 fueron de 202467 millones de dólares (ASEAN Stats, 2020a). Por otra parte, el Sudeste de Asia se consagró como el segundo socio comercial de Beijing durante el primer semestre de 2019, desplazando a Estados Unidos (Xinhua, 2019a).

El stock de inversiones también siguió una tendencia alentadora: en quince años aumentó veintidós veces, revelando la creciente importancia de ASEAN como destino de inversiones chinas (Xinhua, 2019b).

Desde una perspectiva realista de las relaciones internacionales, el principal propósito de la nueva orientación geopolítica de Beijing hacia la ASEAN fue maximizar sus beneficios económicos y geopolíticos a nivel regional y global. En este sentido, el ACFTA parece simbolizar un importante logro en esa dirección (Khan y Yu, 2013). En línea con ello, Beeson (2013) destaca la centralidad del eje estratégico y geoeconómico del acuerdo, más allá de la naturaleza comercial, resaltando que la disposición china de dar a la ASEAN un acceso 
temprano a las ventajas de su mercado es un claro gesto dentro de su "ofensiva del encanto", y de su deseo de garantizar seguridad a sus vecinos, más que una mera iniciativa económica. En otras palabras, la creciente interdependencia económica entre China y los países de ASEAN vuelve cada vez más difícil la idea de prescindir de China, no solo como socio comercial sino como líder regional.

\subsection{La Nueva Ruta de la Seda: El Sudeste de Asia como nodo marítimo en la estrategia geoeconómica de Beijing}

Finalmente, para completar el cuadro de las mayores iniciativas de política exterior de Xi Jinping, la más reciente y de mayor envergadura es la Iniciativa de la Franja y la Ruta (BRI). BRI es un mega plan económico y político y, al mismo tiempo, un emblema de Xi Jinping. Su foco central es la inversión en infraestructura diseñada para "mejorar la conectividad física a lo largo de una ruta de seda marítima y continental” (Mayer, 2018, p. 1228). Según Nolan (2019, p. xxi), Xi Jinping ha hecho de la nueva Ruta de la Seda "una parte clave de las relaciones internacionales de China".

Esta iniciativa se introdujo por primera vez en septiembre de 2013, durante el discurso de Xi en la Universidad Nazarbayev de Kazajstán, donde propuso construir un "Cinturón económico de la Ruta de la Seda". En octubre de 2013, propuso construir una "Ruta de la seda marítima del siglo XXI" durante su discurso en la Cámara de Representantes de Indonesia. Desde una perspectiva de política internacional, la iniciativa materializa un giro del comportamiento internacional chino, pasando del bajo perfil promulgado por Deng Xiaoping a un mayor protagonismo, que la sitúa progresivamente en el centro de la actividad económica global, aumentando su influencia.

A diferencia de las políticas que se explicaron anteriormente, esta iniciativa no solo se concentra en el Sudeste de Asia, aunque sí tiene un especial foco en los países de la región. Este plan de alto nivel es uno de los pilares de la construcción de la "comunidad de destino común" en Asia. Conforme con los objetivos chinos, se logrará a través de una doble vía: profundizando en la integración e interconexión de las economías asiáticas y promoviendo un esquema panasiático de seguridad regional (Delage, 2015). Por otra parte, implicaría la reestructuración de la economía euroasiática y la reconstrucción del núcleo geopolítico de poder chino, a través de la conectividad económica: "mutua conectividad y mutua comunicación” (Soong, 2016, p. 396).

En cuanto a su diseño y operatividad, los corredores son las piezas clave de BRI. Respecto al Sudeste de Asia, el Corredor Económico China-Península 
de Indochina se compone por los principales países integrantes de la ASEAN que, en conjunto, albergan a más de 600 millones de habitantes y que conectarán la ciudad china de Kunming con Singapur a través de puertos y trenes de alta velocidad. Para autores como Johnson (2014), desde el proceso de reformas iniciado con Deng, la política exterior china (incluida aquella dirigida al Este asiático) ha estado subordinada al desarrollo interno y modernización. La iniciativa BRI obedece a esta lógica.

La diplomacia de la periferia, tendiente a incrementar los lazos económicos con los países vecinos, ha generado desconfianza, pues, si bien el crecimiento chino genera prosperidad en la región, también aumenta las dudas sobre la competencia y la excesiva dependencia respecto de ella. La dependencia económica del ASEAN respecto de China es evidente, si se observan las cifras comerciales: en 2017 China concentró el 14,1 \% de las exportaciones del ASEAN y el $20 \%$ de sus importaciones, consolidándose como el principal socio comercial de la región. La participación de China en el comercio exterior del ASEAN ha crecido continuamente en la última década; en 2010 el comercio del ASEAN con China representó el 11,7\% de su comercio total, mientras que en 2017 esta cifra subió al $17,1 \%$. A pesar de que China ha sido un motor para las economías de toda la región, estimulando las exportaciones de bienes y servicios, la balanza comercial es cada vez más negativa para el ASEAN. Aunque la cifra más baja se alcanzó en 2016, el déficit comercial para el ASEAN fue de 68000 millones de dólares en 2017, de 87000 millones de dólares en 2018 y de 103000 millones de dólares en 2019 (Asean Stats, 2020; ITC, 2019).

En cuanto a las inversiones, el principal foco de la iniciativa, los montos han sido incrementales en los últimos años, particularmente en algunos países de ASEAN. Según las cifras oficiales, la inversión extranjera directa (IED) china en ASEAN representó el $3 \%$ del total de las inversiones en la región en 2010; esta proporción casi se triplicó en 2017, y llegó a representar el 8,3\%. Los principales destinos fueron Singapur, Indonesia y Malasia, que representaron el $70 \%$ del total de la IED china en la región. La República Democrática Popular Lao y Vietnam también fueron importantes receptores de inversiones. Para el primero, la IED china representó más del $90 \%$ del total de las entradas de inversiones en el país en 2017, lo que ha generado una dependencia abrumadora de los recursos financieros de Beijing.

Las inversiones chinas también han suscitado preocupación en los países receptores por las consecuencias socioeconómicas -bajos salarios, incentivos a la inmigración ilegal, explotación de trabajadores locales, entre otros- y las condiciones ambientales negativas que suelen estar relacionadas con estos proyectos (Das, 2018; Xue, 2019). Junto con las instituciones e iniciativas multilaterales 
que China primero acompañó y luego fortaleció, la BRI se inscribe en lo que Callahan (2016) identifica como una estrategia más amplia de Beijing de tejer una red sinocéntrica de relaciones económicas, políticas, culturales y de seguridad en su periferia.

A modo de síntesis, se puede señalar que la región aparece -en los tres casos analizados- como una plataforma de proyección global para China, donde el Sudeste de Asia se convierte en un escenario de ensayo de estrategias multilaterales de menor alcance. Todas ellas dan cuenta de un compromiso creciente y paulatino con la estabilidad y crecimiento económico de la región, que se agregan como ladrillos en la construcción de la estrategia de acercamiento económico de Beijing a su periferia y, paralelamente, al globo.

\section{Conclusiones}

Desde 1978, el objetivo de desarrollo económico de China ha generado la necesidad de un sistema internacional pacífico, así como de relaciones de cooperación que posibiliten el sostenimiento del crecimiento económico chino de largo plazo. La crisis de Tiananmen obligó a China a mirar hacia su región para evitar el aislamiento. Más tarde, la crisis de 1997 funcionó como detonante de la integración económica en Asia Oriental, lo cual favoreció un cambio de perspectiva sobre el rol de la cooperación en el mantenimiento de la estabilidad económica. A partir de entonces, Beijing apostó por el diseño de estrategias multilaterales de política exterior en materia económica, algunas destinadas exclusivamente al Sudeste de Asia (ASEAN+3 y ACFTA) y otras que incluyen la participación de espacios geográficos diversos, a la vez que responden a una estrategia global de Beijing (BRI).

La dimensión política-estratégica ha cobrado un renovado vigor, en virtud de la consolidación de su estatus como potencia global y del creciente nacionalismo de la elite gobernante, que le permite una actitud más asertiva ante las crisis internacionales, especialmente aquellas relativas a las disputas territoriales del Mar de China Meridional. No obstante, la dimensión económica continúa siendo central en las relaciones con el Sudeste de Asia, si se considera el objetivo de largo plazo de construir una "comunidad de destino" compartido sinocéntrica en Asia. Y es allí donde China apuesta a las consignas de beneficios mutuos y ganancias compartidas.

Es posible afirmar, entonces, que la estrategia política de Xi Jinping en la región tiene un doble anclaje que se retroalimenta: estimulando la cooperación económica, China puede disminuir las preocupaciones de ASEAN respecto de 
su crecimiento y, profundizando los vínculos económicos, puede también alejar a los países de la región de las posturas belicistas respecto de los problemas territoriales. De esta forma, es posible limitar el comportamiento de las contrapartes en el plano estratégico regional y, paralelamente, fortalecer los lazos económicos. En este sentido, no se observa un quiebre en la política exterior de Xi Jinping, sino que profundiza estrategias de acercamiento regional de sus antecesores, a la vez que muestra una faceta más reivindicativa de aquello que define como interés nacional. Es decir, aquello que China no está dispuesta a negociar.

Si como sostiene Yan (2014), la construcción del liderazgo tiene como condición contar con una autoridad humana o credibilidad estratégica que implica tener cada vez más Estados que legitimen su poderío internacional y apoyen sus políticas, es posible que veamos en un futuro una continuidad de la estrategia China de privilegiar los incentivos antes que las amenazas en su política regional. Esta estrategia le permitirá también consolidar un poder normativo (Callahan, 2016) -a partir de las instituciones e iniciativas regionales- que establezca las reglas del juego para la gobernanza global.

\section{Referencias}

Arase, D. (2016). The geopolitics of Xi Jinping's Chinese dream: problems and prospects. ISEAS-Yusof Ishak Institute.

ASEAN. (2012, 12 de octubre). Declaration on the conduct of the Parties in the South China Sea. https://asean.org/?static_post=declaration-on-the-conduct-of-parties-in-the-south-china-sea-2

ASEAN-China Center. (2013, 2 de octubre). Speech by Chinese President Xi Jinping to Indonesian Parliament. http://www.asean-china-center.org/english/201310/03/c 133062675.htm

ASEAN Secretariat Information Paper. (2017). Overview of ASEAN Plus Three Cooperation. https://asean.org/storage/2017/06/Overview-of-APT-Cooperation-Jun-2017.pdf

ASEAN Stats. (2020). ASEAN Stats Data Portal. https://data.aseanstats.org/

$\mathrm{Ba}, \mathrm{A}$. (2014). Is China leading? China, Southeast Asia and East Asian integration. Political Science, 66(2), 143-165.

Baviera, A. (2016). China's strategic foreign initiatives under Xi Jinping. China Quarterly of International Strategic Studies, 2(1), 57-79. 
Beeson, M. (2013). Living with Giants: ASEAN and the Evolution of Asian Regionalism. TRaNS: Trans -Regional and-National Studies of Southeast Asia, 1(2), 303-322.

Cai, K. (2003). The ASEAN-China free trade agreement and East Asian regional grouping. Contemporary Southeast Asia, 25(3), 387-404.

Callahan, W. (2016). China's “Asia Dream.” Asian Journal of Comparative Politics, $1(3), 226-243$.

Chang Liao, N. (2018). Domestic Politics and China's Assertive Foreign Policy: Why China's Rise May Not Be Peaceful. En T. Leng y R. Aoyama (Eds.), Decoding the Rise of China (pp. 85-106). Palgrave Macmillan.

Chen, D., Pu, X., y Jonhston, A. I. (2014). Debating China's Assertiveness. International Security, 38(3), 176-183.

Christensen, T. (2012). The Advantages of an Assertive China: Responding to Beijing's Abrasive Diplomacy. Foreign Affairs, 90(2), 54-67.

Clinton, H.(2010,23 dejulio). Remarks at the ARF Meeting. NationalConvention Center Hanoi, Vietnam. https://20092017.state.gov/secretary/20092013clinton/ rm/2010/07/145095.htm

Dai Binguo (2010, 6 de diciembre). Adhere To The Path Of Peaceful Development. http://china.usc.edu/dai-bingguo-\%E2\%80\%9Cadhere-path-peacefuldevelopment $\%$ E2\%80\%9D-dec-6-2010

Das, S. (2018). Do the economic ties between ASEAN and China affect their strategic partnership? ISEAS Perspective, 32. Yusof-Ishak Institute.

Delage, F. (2013). La República Popular China y la reconfiguración del orden asiático (1997-2005). Universidad Complutense E-Prints. http://eprints.ucm. es/24641/1/T35175.pdf.

Delage, F. (2015). La estrategia asiática de Xi Jinping. Revista del Instituto Español de Estudios Estratégicos, 5, 1-36.

Fravel, M. (2011). China's Strategy in the South China Sea. Contemporary Southeast Asia 33(3), 292-319.

Friedberg, A. (2015). The Debate Over US China Strategy. Survival, 57(3), 89-110.

Greitens, S. (2013, 11 de junio). US-China relations and America's alliances in Asia. Brooking Opinion. https://www.brookings.edu/ opinions/u-s-china-relations-and-americas-alliances-in-asia/

González Saez, R. (2017, 25 de junio). XXXI Cumbre de ASEAN: La brújula se mueve haciaChina.ObservatoriodelaPolíticaChina.https://politica-china.org/areas/ politica-exterior/xxxi-cumbre-de-asean-la-brujula-se-mueve-hacia-china 
Haacke, J. (2002). Seeking influence: China's diplomacy towards ASEAN after the Asian crisis. Asian perspective, 26(4), 13-52.

Hernández Pedraza, G. (2005). Evolución actual y perspectivas de las relaciones China-ASEAN. En La economía mundial y América Latina. Tendencias, problemas y desafios (pp. 107-136). Consejo Latinoamericano de Ciencias Sociales. http://biblioteca.clacso.edu.ar/clacso/gt/20101013123724/5ParteI4. pdf

Hiep, L. H. (2015)- Vietnam's Alliance Politics in the South China Sea. Trends of Southeast Asia 6, ISEAS, 1-27.

Hoang, T. (2019). From Declaration to Code: Continuity and Change in China's Engagement with ASEAN on the South China Sea. Trends in Southeast Asia. ISEAS.

International Trade Center [ITC] (2019). Trade Map. https://www.trademap.org/

Johnston, A. (2013). How New and Assertive Is China's New Assertiveness? International Security, 37(4), 7-48.

Khan, S. y Yu, L. (2013). Evolving China-ASEAN Relations and CAFTA: Chinese Perspectives on China's Initiatives in Relation to ASEAN Plus 1. European Journal of East Asian Studies, 12, 81-107.

Kissinger, H. (2012). China. Debate.

Kraft, H. J. S. (2017). Great Power Dynamics and the Waning of ASEAN Centrality in Regional Security. Asian Politics \& Policy, 9(4), 597-612.

Kuik, C. (2005). Multilateralism in China's ASEAN policy: its evolution, characteristics, and aspiration 2006. Contemporary Southeast Asia, 27(1), 102-122.

La Voz de Vietnam. (2020, 14 de abril). Concluye la Cumbre Especial de la Asean+3 sobre el Covid-19. https://vovworld.vn/es-ES/noticias/concluye-lacumbre-especial-de-la-asean3-sobre-el-covid19-848387.vov

Leifer, M. (1994). Expanding horizons in Southeast Asia? In Southeast Asian affairs 1994 (pp. 3-21). Institute for Southeast Asian Studies.

Leifer, M. (1995). Dictionary of the modern politics of South-east Asia. Routledge.

Li, M. (2009). Cooperation for Competition: China's Approach to Regional Security in East Asia. Panorama: Insights into Asian and European Affairs, 2, 121-134.

Lye, L. F. y Ha, H. H. (2018). Vietnam's responses to China's maritime assertiveness in the South China Sea. Perspective, 50.

Mayer, M. (2018). China's historical statecraft and the return of history. International Affairs, 94(6),1217-1236. 
Mearsheimer, J (2014, 25 de octubre). Can China Rise Peacefully? The National Interest. https:/nationalinterest.org/print/commentary/ can-china-rise-peacefully-10204

Miks, J. (2012, 11 de abril). China, Philippines in Standoff. The Diplomat. https:// thediplomat.com/2012/04/china-philippines-in-standoff/

Nie, W. (2016). Xi Jinping's foreign policy dilemma. Contemporary Southeast Asia, $38(3), 422-444$.

Nolan, P. (2019). "Preface”. En C. Fang, y P. Nolan, (Eds.), Routledge Handbook of the Belt and Road (pp. xxi-xxiv). Routledge.

Norris, W. (2010). Economic Statecraft with Chinese Characteristics: The Use of Commercial Actors in China's Grand Strategy. Princeton University Press.

Norton, S. (2015). China's grand strategy. China Studies Centre, University of Sydney. http://sydney.edu.au/china_studies_centre/images/content/ccpublications/policy_paper_series/2015/chinas-grand-strategy.pdf

$\mathrm{Pu}, \mathrm{X}$. (2017). Controversial Identity of a Rising China. The Chinese Journal of International Politics, 10(2), 131-149.

Qin, Y. (2014). Continuity through Change: Background Knowledge and China's International Strategy. Chinese Journal of International Politics, 7(3), 285-314

Reilly, J. (2013). China's economic statecraft: turning wealth into power. Analysis. Lowy Institute for International Policy, Sidney. https://www.files.ethz.ch/ isn/175042/reilly_chinas_economic_statecraft_web.pdf

Roberts, C. (2018). The South China Sea Precedent: Rising Instability Amidst Revisionist Tendencies. RSIS Commentaries. Nanyang Technological University, Singapore.

Rocha Pino, M. (2020). La proyección de la Iniciativa Cinturón y Ruta de la Seda en la Unión Europea (2014-2019): Implicaciones económicas y normativas. Relaciones Internacionales, 93(2), 19-46.

Rubiolo, M. F. (2020). The South China Sea Dispute: Reflection of Southeast Asia's Economic and Strategic Dilemmas (2009-2018). Revista Relaciones Internacionales, Estrategia y Seguridad, 15(2), 115-130.

Rubiolo, F. (2010). Política exterior china hacia los procesos de integración regional de Asean: El foro regional de Asean y Asean Plus Three. Astrolabio, 5, 160-184.

Saunders, P. (2013). The Rebalance to Asia: U.S.-China Relations and Regional Security. Strategic Forum, 281, 1-16. 
See Seng, T. (2016). Multilateral Asian Security Architecture: Non-ASEAN Stakeholders. Routledge.

Severino, R. (2010). ASEAN and the South China Sea. Security Challenges, 6(2), $37-47$.

Shih C., y Yin, J. (2013). Between Core National Interest and a Harmonious World: Reconciling Self-role Conceptions in Chinese Foreign Policy. The Chinese Journal of International Politics, 6, 59-84.

Soong, J. (2016) The Political Economy of Development Between China and the ASEAN States: Opportunity and Challenge. The Chinese Economy, 49, 395-399.

Southgate, L. y Khoo, N. (2016). Enter the vanguard state: Reinterpreting ASEAN's response to the South China Sea issue. Journal of Asian Security and International Affairs, 3(2), 221-244.

Storey I. (2018). ASEAN's Failing Grade in the South China Sea. En G. Rozman y J. Liow (Eds.), International Relations and Asia's Southern Tier (pp. 111124). Macmillan Series.

Swaine, M. (2010). Perceptions of an Assertive China. China Leadership Monitor, $32,1-19$.

Vélez Serrano, M. (2018). De dragón revolucionario, a tímido, y ahora rejuvenecido. Relaciones Internacionales, 91(2), 1-23.

Wong, L. (2007). China-ASEAN and Japan-ASEAN relations during the Post-Cold War era. Chinese Journal of International Politics 1, 373-404.

Xi, J. (2017, 3 de noviembre). Informe presentado ante el XIX Congreso Nacional del Partido Comunista Chino. Beijing, China.

Xi, J. (2015, 28 de setiembre). Working Together to Forge a New Partnership of Win-win Cooperation and Create a Community of Shared Future for Mankind. Discurso presentado ante la $70^{\circ}$ sesión de la Asamblea General de Naciones Unidas. Nueva York, Estados Unidos.

Xinhua (2019a, 9 de septiembre). China y ASEAN reforzarán relaciones económicas y comerciales. http://spanish.xinhuanet.com/2019-09/10/c_138379062. htm

Xinhua (2019b, 22 de septiembre). Los lazos entre China y la ASEAN cosechan con visión compartida. https:/www.chinadailyhk.com/articles/179/131/119/1569159780799.html?newsId=106764

Xue Gong. (2019). The Belt \& Road Initiative and China's influence in Southeast Asia, The Pacific Review, 32(4), 635-665. 
Yahuda, M. (2013). China's new assertiveness in the South China Sea. Journal of Contemporary China, 22(81), 446-459.

Yan, X. (2014). From keeping a low profile to striving for achievement. the chinese Journal for International Politics, 7(2), 153-184.

Yang, J. (2010). 'Response and Responsibility: China in East Asian Financial Cooperation', Pacific Review, 23(5), 603-623.

Yang, F. y Li, M. (2016). Comparing China's Claims and Policies in the East and South China Seas: Implications for Regional Security. Journal of Asian Security and International Affairs, 3(2), 135-156.

Zhang, D. (2018). The Concept of 'Community of Common Destiny' in China's Diplomacy: Meaning, Motives and Implications. Asia \& the Pacific Policy Studies 5, 1-12.

Zhang, B. (2015a). Chinese Perceptions of US Return to Southeast Asia and the Prospect of China's Peaceful Rise. Journal of Contemporary China, 24(91), 176-195.

Zhang, C. (2013). Historical Changes in Relations Between China and Neighboring Countries (1949-2012). Asia Paper, Institute for Security and Development Policy.

Zhang, F. (2012). Rethinking China's grand strategy: Beijing's evolving national interests and strategic ideas in the reform era. International Politics, 49(3), $318-345$.

Zhang, J. (2015). China's new foreign policy under Xi Jinping: towards 'Peaceful Rise 2.0'. Global Change. Peace \& Security, 27(1), 5-19.

Zhang, X. (2006). The rise of China and community building in East Asia. Asian Perspective, 30(3), 129-148. 